\title{
Convexly independent subsets of the Minkowski sum of planar point sets
}

\author{
Friedrich Eisenbrand ${ }^{1}$, János Pach ${ }^{2}$, Thomas Rothvoß ${ }^{1}$, and Nir B. Sopher ${ }^{3}$ \\ ${ }^{1}$ Institute of Mathematics, École Polytechnique Féderale de Lausanne, 1015 Lausanne, \\ Switzerland, \{friedrich.eisenbrand,thomas.rothvoss\}@epfl.ch \\ ${ }^{2}$ Courant Institute, NYU and City College, CUNY, USA, pach@cims.nyu.edu \\ ${ }^{3}$ School of Electrical Engineering, Tel-Aviv University, Tel-Aviv 69978, Israel, \\ sopherni@post.tau.ac.il
}

Submitted: Dec 14, 2007; Accepted: Mar 17, 2008; Published: Mar 20, 2008

Mathematics Subject Classification: 52C10, 52A10

\begin{abstract}
Let $P$ and $Q$ be finite sets of points in the plane. In this note we consider the largest cardinality of a subset of the Minkowski sum $S \subseteq P \oplus Q$ which consist of convexly independent points. We show that, if $|P|=m$ and $|Q|=n$ then $|S|=O\left(m^{2 / 3} n^{2 / 3}+m+n\right)$.
\end{abstract}

\section{Introduction}

In connection with a class of convex combinatorial optimization problems (Onn and Rothblum, 2004), Halman et al. (2007) raised the following question. Given a set $X$ of $n$ points in the plane, what is the maximum number of pairs that can be selected from $X$ so that the midpoints of their connecting segments are convexly independent, that is, they form the vertex set of a convex polygon? In the special case when the elements of $X$ themselves are convexly independent, they found a linear upper bound, $5 n-6$, on this quantity. They asked whether there exists a subquadratic upper bound in the general case. In this note, we answer this question in the affirmative by establishing an upper bound of $O\left(n^{4 / 3}\right)$.

We first reformulate the question in a slightly more general form. Let $P$ and $Q$ be sets of size $m$ and $n$ in the plane. The Minkowski sum of $P$ and $Q$ is $P \oplus Q=\{p+q \mid p \in$ $P, q \in Q\}$.

What is the maximum size of a convexly independent subset of $P \oplus Q$ ? 
More precisely, we would like to estimate the function $M(m, n)$, which is the largest cardinality of a convexly independent set $S$, which is a subset of the Minkowski sum of some planar point sets $P$ and $Q$ with $|P|=m$ and $|Q|=n$.

Notice that the set of all midpoints of the connecting segments of an $n$-element set $P$ can be expressed as $\frac{1}{2}(P \oplus P)$, so that $M(n, n)$ is an upper bound on the quantity studied by Halman et al.

Let $S$ be a convexly independent subset of $P \oplus Q$. Consider the bipartite graph $G$ on the vertex set $P \cup Q$, in which $p \in P$ and $q \in Q$ are connected by an edge if and only if $p+q \in S$. It is easy to check that $G$ cannot contain $K_{2,3}$ as a subgraph. Applying the forbidden subgraph theorem (Kövári et al., 1954), see also (Pach and Agarwal, 1995), it follows that $|S|=O(\sqrt{m} \cdot n+m)$.

Our next result provides a better bound.

Theorem 1. Let $P$ and $Q$ be two planar point sets with $|P|=m$ and $|Q|=n$. For any convexly independent subset $S \subseteq P \oplus Q$, we have $|S|=O\left(m^{2 / 3} n^{2 / 3}+m+n\right)$.

\section{Proof of Theorem 1}

We reduce the problem to a point-curve incidence problem in the plane. A closed set $K \subseteq \mathbb{R}^{2}$ is strictly convex, if for each $a, b \in K$ the interior of the line-segment $\operatorname{conv}(\{a, b\})$ is contained in the interior of $K$. A closed curve $C$ is strictly convex if it is the boundary of a strictly convex set. Consider now $n$ translated copies $C+t_{1}, \ldots, C+t_{n}$ of $C$, and $m$ points $p_{1}, \ldots, p_{m}$. Let $I(m, n)$ denote the maximum number of point-curve incidences which occur in such a configuration. Notice that $C+t_{i}$ and $C+t_{j}$ intersect in at most two points for $i \neq j$. Furthermore, for any two distinct points $p_{\mu}$ and $p_{\nu}$, there exist at most two curves $C+t_{i}$ incident to both $p_{\mu}$ and $p_{\nu}$. We can apply the following well known upper bound on the number $I(m, n)$ of incidences between $m$ points and $n$ "well-behaved" curves with the above properties, see (Pach and Sharir, 1998).

$$
I(m, n)=O\left(m^{2 / 3} n^{2 / 3}+m+n\right) .
$$

Thus, to establish Theorem 1, it remains to prove

Theorem 2. For any positive integers $m$ and $n$, we have $M(m, n) \leqslant I(m, n)$.

Proof. Let $P=\left\{p_{1}, \ldots, p_{m}\right\}, Q=\left\{q_{1}, \ldots, q_{n}\right\}$, and assume that $S$ is a convexly independent subset of $P \oplus Q$. Clearly, there is a strictly convex closed curve $C$ passing through all points in $S$. Consider the $n$ translates $C-q_{1}, \ldots, C-q_{n}$ of $C$. Count the number of incidences between these curves and the elements of $P$. Notice that if the point $p+q$ belongs to $S$, then $p$ is incident to $C-q$. Since no two distinct points $p_{1}+q_{1} \neq p_{2}+q_{2} \in S$ are associated with the same incidence, the result follows. 


\section{Unit distances}

Theorem 1 can also be deduced from the known upper bounds on the number of unitdistance pairs induced by $n$ points in a normed (Minkowski) plane. For this, notice that one can replace $C$ by a centrally symmetric strictly convex curve $C^{\prime}$ such that the number $I^{\prime}$ of incidences between the curves $C^{\prime}-q_{1}, \ldots, C^{\prime}-q_{n}$ and the points in $P$ is at least half of the number $I$ of incidences between the curves $C-q_{1}, \ldots, C-q_{n}$ and the points in $P$. The curve $C^{\prime}$ defines a norm, and thus a metric, in the plane, with respect to which the unit circle is a translate of $C^{\prime}$. Therefore, $I^{\prime}$ can be bounded from above by the number of unit-distance pairs between the set of centers of the curves $C^{\prime}-q_{1}, \ldots, C^{\prime}-q_{n}$ and the elements of $P$, which is known to be $O\left(m^{2 / 3} n^{2 / 3}+m+n\right)$.

In particular, for $m=n$, this number cannot exceed the maximum number $u(2 n)$ of unit-distance pairs in a set of $2 n$ points in a normed plane with a strictly convex unit circle. It is known that $u(2 n)=O\left(n^{4 / 3}\right)$ (see e.g. (Brass, 1996)), and a gridlike construction shows that this bound can be attained for certain norms (Brass, 1998; Valtr, 2005). Note that in the Euclidean norm, the number of unit-distance pairs induced by $n$ points is $n e^{\Omega(\log n / \log \log n)}$, and this estimate is conjectured to be not far from best possible (Erdős, 1946).

The question arises whether any of the examples establishing the tightness of the upper bounds on $I(m, n)$ and $u(n)$ can be used to show that Theorem 1 is also optimal. Unfortunately, in all known constructions, most elements of $P \oplus Q$ can be written in the form $p+q(p \in P, q \in Q)$ in many different ways. Therefore, any element of a convexly independent subset of $P \oplus Q$ may be associated with several incidences between a curve $C-q$ and a point of $P$. This suggests that the maximum size of a convexly independent subset of $P \oplus Q$ can be much smaller than $I(m, n)$. For $m=n$, we do not know any example for which $P \oplus Q$ has a convexly independent subset with a superlinear number of elements.

\section{References}

Brass, P. (1996). Erdős distance problems in normed spaces. Computational Geometry. Theory and Applications, 6(4):195-214.

Brass, P. (1998). On convex lattice polyhedra and pseudocircle arrangements. In Charlemagne and his heritage. 1200 years of civilization and science in Europe, Vol. 2 (Aachen, 1995), pages 297-302. Brepols, Turnhout.

Erdős, P. (1946). On sets of distances of $n$ points. The American Mathematical Monthly, $53: 248-250$.

Halman, N., Onn, S., and Rothblum, U. (2007). The convex dimension of a graph. Discrete Applied Mathematics, 155:1373-1383. 
Kővári, T., Sós, V. T., and Turán, P. (1954). On a problem of K. Zarankiewicz. Colloquium Math., 3:50-57.

Onn, S. and Rothblum, U. (2004). Convex combinatorial optimization. Discrete \& Computational Geometry, 32:549-566.

Pach, J. and Agarwal, P. K. (1995). Combinatorial geometry. Wiley-Interscience Publication. New York.

Pach, J. and Sharir, M. (1998). On the number of incidences between points and curves. Combinatorics, Probability \& Computing, 7(1):121-127.

Valtr, P. (2005). Strictly convex norms allowing many unit distances and related touching questions. Manuscript. 